TURKU - FL - R18

\title{
Baryogenesis in the singlet Majoron model
}

\author{
I. Viljađ \\ Department of Physics \\ University of Turku \\ FIN-20500 Turku \\ Finland
}

\begin{abstract}
The baryogenesis by decays of the right-handed neutrinos in the Majoron model is studied. We show that compared to the observational value it is possible to produce large enough baryon asymmetry in the Majoron model or, at least, contribute strongly to it. It requires that the mass of the lightest right-handed neutrino is about (or above) $1 \mathrm{TeV}$ and the self coupling of the singlet sector is weak enough, $<2 \times 10^{-5}$, to prevent the thermalization of the right-handed neutrinos by annihilations.
\end{abstract}

\footnotetext{
${ }^{1}$ internet: vilja@sara.cc.utu.fi
} 
The singlet Majoron model [1] is one of the simplest extensions of the Standard Model explaining many question remained open in the Standanrd Model. In the context of the Majoron model the vanishingly small neutrino masses can be explained by using spontaneous breaking of a global U(1) symmetry and sew-saw mechanism. The right handed neutrinos aquire large Majorana masses while the left handed ones keep very light. On the other hand, in the Standard Model the baryogenesis appears to be difficult to realize [2, 3] due to too small $\mathrm{CP}$-violation and sphaleron [4] mediated $B+L$ violating transitions. Those transitions not only erase any pre-existing baryon asymmetry, but are also able to wash out the asymmetry produced during the electro-weak phase transition. In the matter of fact, the experimental Standard Model higgs mass lower bound $m_{H}>60 \mathrm{GeV}$ [5] indicates that the observed baryon-to-photon ratio $n_{B} / n_{\gamma} \sim 10^{-10}$ is too large to be explained by baryogenesis during the electroweak phase transition of the minimal Standard Model.

It has been proposed that the decay of heavy Majorana neutrinos could be responsible of the baryogenesis [6, 7, 8]. In this scenario the out-off-equilibrium decays of heavy righthanded neutrinos produce a non-zero lepton number $L$ which is converted to $B$ asymmetry by sphaleron mediated transitions. Therefore the singlet Majoron model, as a minimal extension of the Standard Model, is a natural and interesting candidate for explaining both the neutrino masses and the observed baryon asymmetry. The relation between baryogenesis scale and the neutrino masses has also been discussed in the literature [9]. In this paper we study the singlet Majoron model, in particular the decay rate of the heavy right-handed neutrinos and compare it to their annihilation rate showing that for suitable parameters the baryon asymmetry can be produced although it seems to require that the singlet sector is weakly coupled to the the doublet sector. For these purposes we have to study the phase structure of the Majoron model and equilibration of the right-handed neutrinos.

The Majoron model contains, in addition to the Standard Model doublet Higgs $H$, one electroweak singlet field $S$. To the fermionic sector has been added the right-handed neutrinos $N_{i}$, where $i$ is a generation index. Both $S$ and $N_{i}$ are in non-trivial representations of the global U(1) group, whereas other fermions and the Higgs field $H$ are singlets with respect it. The classical potential reads

$$
V_{0}(H, S)=m_{H}^{2}|H|^{2}+m_{S}|S|^{2}+\gamma|H|^{2}|S|^{2}+\beta|S|^{4}+\lambda|H|^{4}
$$

where the mass-like parameters $m_{H}^{2}, m_{S}^{2}<0$ and the couplings $\beta, \lambda>0$ with $\gamma^{2}<4 \lambda \beta$. (See Ref. [10 for detailed study of the potential.) At finite temperature the potential is 
modified at one-loop level (keeping only leading terms) as

$$
V_{T}(H, S)=\mu_{H}^{2}(T)|H|^{2}+\mu_{S}^{2}(T)|S|^{2}+\gamma|S|^{2}|H|^{2}+\beta|S|^{4}+\lambda|H|^{4}-\frac{T}{12 \pi} \operatorname{tr} M^{3}+\ldots
$$

where $M$ is the general scalar mass matrix and the dots stand for remaining finite temperature corrections. The temperature corrected masses introduced in (2) are given by

$$
\begin{aligned}
\mu_{H}^{2}(T) & =m_{H}^{2}+r^{\prime} \frac{T^{2}}{4} \\
\mu_{S}^{2}(T) & =m_{S}^{2}+s^{\prime} \frac{T^{2}}{3}
\end{aligned}
$$

where

$$
\begin{aligned}
r^{\prime} & =\frac{2 m_{W}^{2}+m_{Z}^{2}+2 m_{t}^{2}}{f^{2}} \simeq 0.67, \\
s^{\prime} & =\beta+\frac{1}{8} \gamma+\frac{1}{8} h_{\nu}^{2},
\end{aligned}
$$

$f=247 \mathrm{GeV}$ is the vacuum expectation value of the doublet higgs and $h_{\nu}^{2}$ stands collectively for all right-handed Yukawa couplings: $h_{\nu}^{2}=\sum_{i} h_{i}^{2}$. In the equation (5) we have omitted the scalar contribution to $r^{\prime}$ as small. The cubic term $\operatorname{tr} M^{3}$ is a complicated expression of the fields [10, but can be simplified considerably in some special cases.

To make the baryogenesis possible, the symmetry breaking has to proceed first to the direction of the singlet field $S$, so that only the global U(1) symmetry breaks generating large Majorana masses for the right-handed neutrinos. The ratio of critical temperatures in the directions of $S$ and $H, T_{c, S}$ and $T_{c, H}$, respectively, are given by

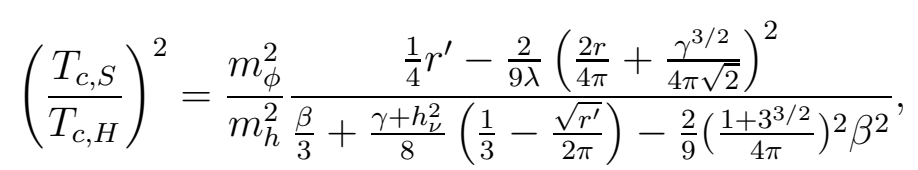

where $r=\frac{2 m_{W}^{3}+m_{Z}^{3}}{f^{3}} \simeq 0.35$. The phase structure of the singlet Majoron model has been extensively analyzed in Ref. [10]. The ratio (7) has to be larger than one in order to have two-stage phase transition proceeding first to the singlet direction. If $\mathrm{U}(1)$-symmetry is broken first, the critical temperature to the doublet direction is

$$
\left(T_{c, H}^{\prime}\right)^{2}=4 \frac{m_{H}^{2}-\frac{\gamma}{2 \beta} m_{S}^{2}}{r^{\prime}-\frac{r^{2}}{2 \pi^{2} \lambda}} .
$$

It is of essential importance that the right-handed neutrinos have also obtained masses before the sphaleron transitions freeze out at $T_{s p h}$, i.e. before they become too ineffective to 
convert any lepton number to baryon number. We require that the mass $m_{N_{1}}=h_{1} \bar{f}$ of the lightest right-handed neutrino is larger than $T_{s p h}$ given by solving the equation [3]

$$
E_{s p h}^{S M}\left(T_{s p h}\right) / T_{s p h} \equiv x \simeq 45
$$

where $E_{s p h}^{S M}(T)=4 \pi B(\lambda) f(T) / g_{W}$ is the Standard Model sphaleron energy, $g_{W}^{2}=0.436$ and $B(\lambda)$ is a smooth, increasing function varying between values 1.56 and 2.72. Although Eq. (9) defines the freeze-out temperature for the Standard Model, it is with high accurary same than in the Majoron model [11]. Thus $\frac{m_{N_{1}}}{T_{s p h}}>1$ is a necessary condition for baryogenesis via neutrino decay. It can be cast in the form

$$
1<\frac{m_{N_{1}}^{2}}{T_{s p h}^{2}}=\left(\frac{2 r}{4 \pi}\right)^{2}\left[\left(\frac{2 x g_{W}}{3 r B(\lambda)}\right)^{2} \lambda+r^{\prime}\left(\frac{4 \pi}{3 r}\right)^{2}-\frac{4 x g_{W}}{3 r B(\lambda)}\right] \frac{4 \beta}{4 \beta \lambda-\gamma^{2}} \frac{m_{N_{1}}^{2}}{f^{2}} .
$$

The decay rate of the lightest right-handed neutrino (chosen to be the first generation neutrino) is given by

$$
\Gamma_{d}=\frac{1}{8 \pi^{2}} \frac{m_{d_{1}}^{2}}{f^{2}} m_{N_{1}}
$$

where $m_{d_{1}}$ is the Dirac mass of the lightest generation, $m_{d_{1}} \simeq m_{e} \sim 1 \mathrm{MeV}$. This rate has to be compared to the expansion rate of the universe, to the Hubble rate $H$ :

$$
H(T)=1.66 g_{*}^{1 / 2} \frac{T^{2}}{M_{P l}}
$$

where $g_{*}$ is the effective number of degrees of freedom, $g_{*}=110.5$ (at $T \sim m_{N_{1}}$ ), and $M_{P l} \simeq 1.210^{19} \mathrm{GeV}$ is the Planck mass. A condition for out-of-equilibrium decay can be written now as

$$
\Gamma_{d}<2 H
$$

ehich has to be evaluated at $T \sim m_{N_{1}}$. This is, however, not a sufficient condition, but one has to take accout that the annihilations $N N \rightarrow \chi \rho$ and $N N \rightarrow \chi \chi$, where $\chi$ is the Majoron and $\rho$ is the massive part of the singlet scalar, tend to keep the right-handed neutrinos in thermal equilibrium. The thermally averaged annihilation rates are approximately given by

$$
\Gamma_{a n n}=\left\langle\sigma v_{r e l}\right\rangle n(T) \simeq \frac{\beta^{2}}{32 \pi}\left(1-\frac{m_{\rho}^{2}}{4 m_{N}^{2}}\right)^{ \pm 1} \frac{n(T)}{m_{N}^{2}}
$$

where $m_{\rho}^{2}=2 \beta \bar{f}^{2}$ is the singlet scalar mass before electroweak breaking and $n(T)$ is the usual number density of the right-handed neutrinos. The upper sign refers to the process $N N \rightarrow$ $\chi \rho$ whereas the lower sign refers to the process $N N \rightarrow \chi \chi$. At the relevant temperature, 
$T \sim m_{N_{1}}$, the annihilation rate is $\Gamma_{a n n} \simeq 4.610^{-4} \beta^{2} m_{N_{1}}\left(1-\frac{m_{\rho}^{2}}{4 m_{N_{1}}}\right)^{ \pm 1}$. We require that the annihilations must proceed much slower than the decays, i.e. $\Gamma_{a n n} \ll \Gamma_{d}$. Explicitely written the condition reads

$$
\beta\left(1-\frac{\beta}{2 h_{1}^{2}}\right)^{ \pm 1 / 2} \ll 2 \times 10^{-5} .
$$

We have got three necessary conditions (10), (13) and (15) for non-equilibrium decays of the right-handed neutrinos. It as fairly easy to see that the condition (10) is practically allways satisfied if the condition (13) is, and the condition (15) implies $\beta \ll 2 \times 10^{-5}$. On the other hand in order to have that small value of $\beta$ stable against radiative effects, in particular the correction from $H$-loop has to be small enough, i.e. $\frac{\gamma^{2}}{64 \pi^{2}} \ll 2 \times 10^{-5}$. This yields an upper bound $\gamma \ll 0.12$. In practise, however, this bound is not very restictive, but the stability bound of the potential, $\gamma^{2}<4 \lambda \beta$, is much stricter.

The analysis above shows, when the enviroment for the baryon asymmetry generation is favorable, but it does not tell anything precise about the net baryon number $N_{B}=n_{B} / n_{\gamma}$ produced in the neutrino decays. For that purpose one has to calculate first the $B-L$ number, $N_{B-L}$, produced by decays of the right-handed neutrinos. This can be done by solving the relevant Boltzman equation, yielding [12]

$$
N_{B-L} \simeq \frac{0.3 \epsilon}{g_{*} K(\ln K)^{0.6}},
$$

where

$$
\begin{aligned}
K & =\left.\frac{\Gamma_{d}(T)}{2 H(T)}\right|_{T=m_{N_{1}}}, \\
\epsilon & \simeq \frac{1}{\pi} \frac{m_{D_{3}}^{2}}{f^{2}} \frac{m_{N_{1}}}{m_{N_{3}}} \delta
\end{aligned}
$$

and $\delta$ is the $\mathrm{CP}$-violating phase. Here is assumed that $h_{3} \gg h_{2} \gg h_{1}$, i.e. the third generation is the heaviest and the first generation is the lightest one. Afterwards the lepton number $N_{L}$ (= $N_{B-L}$ here) is convereted to the baryon number $N_{B}$ by the sphaleron mediated transitions according to [7]

$$
N_{B}=\frac{28}{79} N_{B-L}
$$

To get some idea about the magnitude of the baryon number generated, we use values like $m_{D_{1}} \sim 1 \mathrm{MeV}, m_{D_{3}} \sim 1 \mathrm{GeV}, m_{N_{1}} \sim 1 \mathrm{TeV}$ and $\frac{m_{N_{1}}}{m_{N_{3}}} \delta \sim 10^{-1}$. We obtain $N_{B} \sim$ $10^{-10}-10^{-12}$ and clearly the right-handed neutrino decays are at least contributing to the baryon number of the universe. It is, however, clear that the calculation contains many 
uncertainties, primarily due to uncertainties on neutrino masses. In particular, if $m_{N_{1}}<1$ $\mathrm{TeV}$, the right-handed neutrino contribution would be smaller than $10^{-12}$. On the other hand, if $m_{N_{1}}$ were larger than few TeV's, the appealing feature of the dynamical contact between $\mathrm{U}(1)$ breaking and electroweak breaking would be lost, unless the paremeters of the potential are suitably fine-tuned [10]. The result of the present paper, together with other baryogenesis results of the singlet Majoron model [10], suggests anyway that the observed baryon asymmetry is, perhaps, not a result of a single baryogenesis mechanism but might be produced as result of several physical phenomena. 


\section{References}

[1] Y. Chikashige, R.M. Mohapatra and R.D. Peccei, Phys. Lett. 98 (1981) 265.

[2] V.A. Kuzmin, V.A. Rubakov and M.E. Shaposnikov, Phys. Lett. B155 (1985) 36.

[3] M.E. Shaposnikov, JETP Lett. 44 (1986) 465; Nucl. Phys. B287 (1987) 757.

[4] F.R. Klinkhamer and N.S. Manton, Phys. Rev. D30 (1984) 2212.

[5] T. Mori, Proceedings of the XXVI International Conference on High Energy Physics, 1992, Dallas.

[6] M. Fukugita and T. Yanagida, Phys. Lett. B174 (1986) 45; A.E. Cohen, D.B. Kaplan and A.N. Nelson, Nucl. Phys. B349 (1991) 727.

[7] C.E. Vayonakis, Phys. Lett. B286 (1992) 92.

[8] K. Enqvist and I. Vilja, Phys. Lett. B299 (1993) 281.

[9] M. Fukugita and T. Yanagida, Phys. Rev. D42 (1990) 42; A.N. Nelson and S.M. Barr, Phys. Lett. B246 (1990) 141; W. Fischler et al., Phys. Lett. B258 (1991) 45; J.A. Harvey and M.S. Turner, Phys. Rev. D42 (1990) 3344.

[10] K. Enqvist, K. Kainulainen and I. Vilja, Nucl. Phys. B403 (1993) 749.

[11] K. Enqvist and I. Vilja, Phys. Lett. B287 (1992) 119.

[12] E.W. Kolb and M.S. Turner, The Early Universe, Addison-Wesley, Rearding, MA, 1990. 